\title{
Stellar and Galactic studies with the 2-m Himalayan Chandra Telescope
}

\author{
Aruna Goswami \\ Indian Institute of Astrophysics, Koramangala 2nd Block, Bengaluru, India, 560034
}

\begin{abstract}
The Himalayan Chandra Telescope (HCT), of the Indian Astronomical Observatory (IAO), is currently one of the most heavily subscribed telescopes in India. The telescope has been in regular use for scientific observations by national as well as international astronomical communities since May 2003. Over the past fifteen years, various observational programs have been carried out using HCT contributing to our understanding of many different aspects of stellar and Galactic astronomy. Here, a glimpse of spectroscopic studies made using HCT is provided with an aim to expand potential collaboration in the area of stellar and Galactic studies using these observing facilities. Some results from our long-term observing program on a search for $\mathrm{CH}$ stars from low resolution spectroscopy of high Galactic latitude objects that has given us the potential to discover objects of rare types are discussed.
\end{abstract}

Keywords: stars: carbon - stars: classification - stars: s-process elements

\section{Introduction}

HCT is a 2-m aperture optical-infrared telescope at the Indian Astronomical Observatory (IAO), Hanle. It is remotely operated from the Centre for Research \& Education in Science \& Technology (CREST), Hosakote via a dedicated satellite link. The telescope was released for science observations in May 2003. Currently, the telescope is equipped with three science instruments, namely, the Himalaya Faint Object Spectrograph (HFOSC), the TIFR near-infrared Imaging Spectrograph (TIRSPEC), and the Hanle Echelle Spectrograph (HESP). These intruments are mounted on an instrument mount cube at the cassegrain focus of the telescope that has four side ports and an on-axis port, which makes all three instruments available mounted on the telescope ${ }^{1}$. HFOSC, is an optical imager cum spectrograph. It is possible to shift between the imaging and spectroscopic modes of operation in seconds. TIRSPEC, mounted on HCT covers wavelength from 1 to 2.5 micron, with a resolution of $\sim 1200$, for near-infrared medium resolution spectroscopy. The instrument provides a Field of View (FoV) of $307 \times 307 \operatorname{arcsec}^{2}$ in imaging mode. In spectroscopy mode, the instrument can be used in single order mode to cover 1.02-1.20 micron, 1.21-1.48 micron, 1.49-1.78 micron and 2.04-2.35 micron. In cross disperse modes, the instrument provides simultaneous coverage of 1.02-1.49 micron and 1.50-2.45 micron ${ }^{2}$. The instrument is sensitive to low temperature stellar photospheres $(\mathrm{T} \leq 2500$ $\mathrm{K}$ ) and objects surrounded by warm dust envelopes or embedded in dust or molecular clouds. It is

\footnotetext{
${ }^{1}$ (https://www.iiap.res.in/?q=telescope_iao)

${ }^{2}$ (https:// web.tifr.res.in/ daa/tirspec/)
} 
particularly suited for searching for low-mass stellar populations (M, L dwarfs, brown dwarfs), strong mass-losing stars on the asymptotic giant branch, young stellar objects still in their protostellar envelopes and active galactic nuclei (Ojha et al. 2012). HESP is a fibre-fed, high resolution $(\mathrm{R}=30,000$ and 60,000) spectrograph, the wavelength coverage spans from 350 to $1000 \mathrm{~nm}$. The instrument is well suited for chemical composition studies of stars belonging to different evolutionary stages that are brighter than $\mathrm{V}_{\text {mag }} \sim 13$. The high mechanical stability and double fibre mode allow measurement of precise radial velocities, essential for programs such as exoplanet studies and asteroseismology ${ }^{3}$.

All the three instruments are used regularly to address a variety of challenging astrophysical problems. While HFOSC is the first light instrument, TIRSPEC was made available for observations since May 2014, and the HESP from January 2017. Understanding stellar nucleosynthesis, and chemical and dynamical evolution of our Galaxy is one of the primary areas of interest, and several on-going programs at HCT use low as well as high resolution spectroscopy to study stars belonging to different evolutionary stages: F-G supergiants, metal-poor stars, Li-rich stars, K-giants, CH stars, barium stars, carbon stars as well as hydrogen deficient carbon stars etc.

Standard stellar evolutionary models predict severe depletion of surface Li abundance, which is as low as 1.4 dex in K giants (Iben 1967), a factor of about 80 lower than the maximum value of about 3.3 dex observed in main sequence stars. Contrary to predictions and general observational trends the discovery of high $\mathrm{Li}$ abundance $(\mathrm{A}(\mathrm{Li})=2.95)$, in a normal $\mathrm{K}$ giant (Wallerstein and Sneden 1982) naturally raised questions regarding the formation and evolution of $\mathrm{Li}$ in $\mathrm{RGB}$ stars, that still remain poorly understood. Since then, more Li-rich giants have been discovered. In an observing program with HFOSC about $2000 \mathrm{~K}$ giants were subjected to low resolution spectroscopy, and about $15 \mathrm{Li}$-rich stars were detected (Kumar et al. 2015). The sample contained stars in the H-R diagram, along the RGB starting from the first dredge-up to well above the luminosity bump.

Observation of R Coronae Borealis type stars (RCBs), Dy Per like stars and extreme helium stars (EHes) is another long term observing program with HCT. These objects are low mass hydrogen deficient supergiants associated with very late stages of stellar evolution. Understanding evolutionary connection between RCBs, DY Pers, EHes, and HdCs still remains an outstanding problem. ${ }^{18} \mathrm{O}$ is highly enhanced and ${ }^{13} \mathrm{C}$ is negligible in RCBs and HdCs than in normal carbon stars, and these are clues to their origin and evolution. Using TIRSPEC, from ${ }^{12} \mathrm{C}^{18} \mathrm{O}$ and ${ }^{13} \mathrm{C}^{18} \mathrm{O}$ molecular bands observed in K-band, the isotopic ratios measured on the spectra of DY Per candidates were found to be consistent with that of RCB and HdC stars indicating that DY Per type stars could be related to the $\mathrm{RCB} / \mathrm{HdC}$ class of stars (Bhowmick et al. 2018).

Observational programs with HCT also include studies on characteristic properties of molecular clouds, star forming regions, diffuse interstellar matter, Young Stellar Objects, chromospherically active stars, and Planetary Nebulae that provide insight and better understanding of star formation. Finding new variable star candidates from open clusters and Globular clusters, using both photometry and spectroscopy are also long-term observational programs. Over the last ten years or so, timeseries CCD observations of a family of selected Globular Clusters north of -20 degrees, have led to the discovery of many new variables of several types RR Lyrae, SX Phe, anomalous Cepheids, binary stars, and large populations of Blazhko variables even among the RRc stars (A. A. Ferro and collaborators).

There are also a few observing programs with primary objectives to detect exoplanets, monitoring transiting exoplanets, and studying characteristic properties of stars with planets, and low-mass eclipsing binaries using HESP. Time domain astronomy with HCT includes IR and optical followup studies on novae and cataclysmic variables, symbiotic stars, supernovae, and gamma-ray burst sources. In the following, we will discuss our long term observational program with HCT using HFOSC on a search for $\mathrm{CH}$ stars. Some results from this program have been already published in our

\footnotetext{
${ }^{3}$ (https:// www.iiap.res.in/?q=hanle_echelle_spectrograph)
} 
earlier papers (Goswami 2005, Goswami et al. 2007, 2010a); here we summarize the main results and present a compilation of the potential $\mathrm{CH}$ star candidates including the new detections made after these works (Table 1).

\section{Why search for CH stars}

$\mathrm{CH}$ stars characterized by iron-deficiency and carbon enhancement (with $-2 \leq[\mathrm{Fe} / \mathrm{H}] \leq-1$, $[\mathrm{C} / \mathrm{Fe}]$ $\geq 0.7,[\mathrm{Ba} / \mathrm{Fe}] \geq 1.0$ ) are a distinct group of stars (Keenan 1942) that are long been used as halo tracers. CEMP-s stars (Beers \& Christlieb 2005) with $[\mathrm{C} / \mathrm{Fe}]>1$ and $[\mathrm{Fe} / \mathrm{H}]<-2$ that also show s-process enhancement are more metal-poor counterparts of $\mathrm{CH}$ stars and believed to be extrinsically enriched with carbon and s-process elements. Chemical composition studies of a number of these objects have demonstrated that significant insight into the neutron-capture processes taking place in the early Galaxy can be derived from these objects (Norris et al. 1997a 1997b, 2002; Bonifacio et al. 1998; Hill et al. 2000; Aoki et al. 2002; Goswami et al. 2006, 2016, Aoki et al. 2007). These stars characterized by strong G-band of $\mathrm{CH}$, and $s$-process elements play significant roles in probing the impact of $s$-process mechanisms in early Galactic Chemical Evolution (GCE), providing insight into the time of influence of this process on early GCE. Nucleosynthesis theories do not support the occurrence of s-process nucleosynthesis during the stellar evolutionary phases to which these stars belong. $\mathrm{CH}$ stars are found to be as binaries with a now invisible white dwarf companion (McClure et al. 1980, McClure 1983, 1984, McClure \& Woodsworth 1990) showing radial velocity variability. These stars are believed to have received, via binary mass transfer mechanisms, the products of the companion stars produced during their AGB phase of evolution. Hence, the chemical composition of this class of objects can be used to trace the AGB nucleosynthesis at low-metallicity. However, high-resolution spectroscopic analysis for a large sample of these stars is needed to understand the distribution of neutron-capture elements with respect to metallicity and their contribution to the Galactic chemical enrichment. As the metallicity decreases, the number of seed nuclei for neutron-capture decreases, and this favours the production of heavy s-process elements, increasing the [hs/ls] ratio, where hs refers to the heavy s-process elements and ls refers to the light s-process elements. Hence, the [hs/ls] ratio could provide clues to the origin of the s-process nucleosynthesis, the reactions operating at different metallicities and hence to the chemical enrichment of the Galaxy. Accurate identification and spectroscopic characterization of $\mathrm{CH}$ stars are therefore very essential. However, literature survey shows that not many $\mathrm{CH}$ stars have been studied in detail. The main difficulty lies in distinguishing these objects from other carbon stars, such as Pop I C-R and C-N stars. At low resolution, the dwarf carbon stars also exhibit remarkably similar spectra to those of $\mathrm{C}$ giants.

\section{Selection of program stars}

Program stars are chosen from the list of Faint High Latitude Carbon (FHLC) stars of Christlieb et al. (2001) that contains a sample of 403 stars. Some field stars are also included based on their high proper motion estimates. The identification of FHLC stars was based on a measure of line indices - i.e. ratios of the mean photographic densities in the carbon molecular absorption features and the continuum band passes. They primarily considered strong $\mathrm{C}_{2}$ and $\mathrm{CN}$ molecular bands shortward of $5200 \AA$ but not the $\mathrm{CH}$ bands. We have undertaken to search for $\mathrm{CH}$ stars in this sample based on a

set of well defined spectral criteria (section 5). We have selected objects with $\mathrm{V}_{\text {mag }} \leq 16$ and limited by declination -26 degree. 


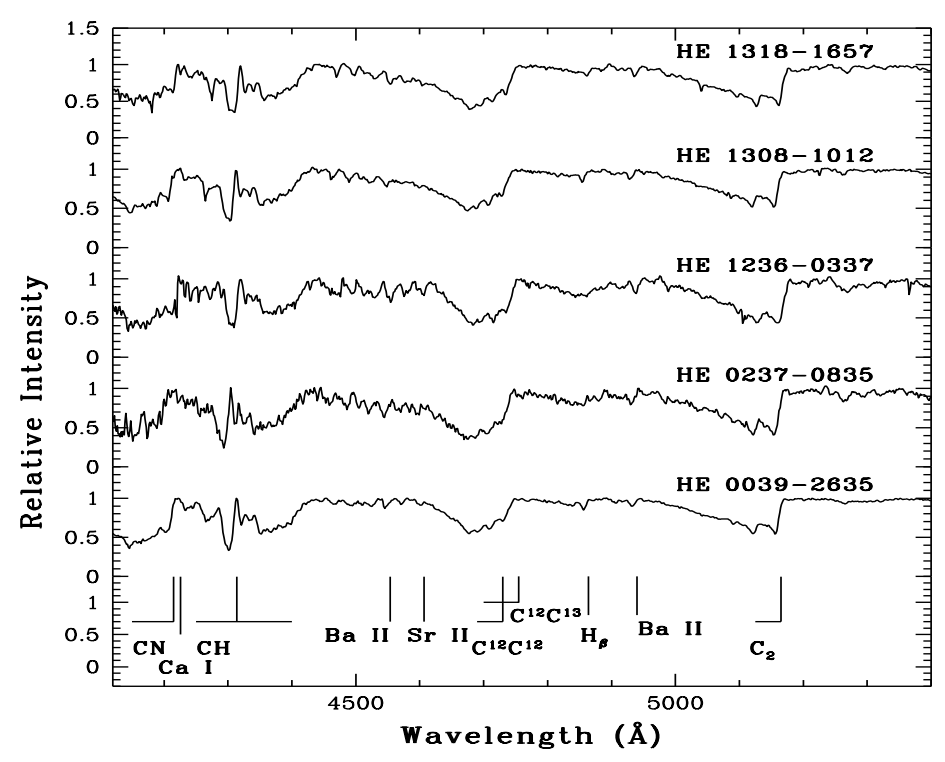

Figure 1: Sample spectra of CH stars in the wavelength region $4100-5400 \AA$. Prominent features seen on the spectra are indicated.

\section{Observation and Data Reduction}

The stars are observed with 2-m HCT using HFOSC. Spectra of a number of stars such as HD 182040, HD 26, HD 5223, HD 209621, Z PSc, V460 Cyg and RV Sct that belong to different classes of carbon stars are also taken for a comparison. The grism and the camera combination used for observation provided a spectral resolution of $\sim 1330(\lambda / \delta \lambda)$; the observed bandpass ran from about 3800 to 6800 $\AA$. Observations of Fe-Ar hollow cathod lamp provided the wavelength calibration. Data reduction is carried out using the IRAF ${ }^{4}$ software spectroscopic reduction packages. A few sample spectra of $\mathrm{CH}$ stars are shown in Figure 1.

\section{$5 \quad$ Spectral analysis and Results}

To establish the membership of a star in a particular group we have conducted a differential analysis of the program stars spectra with the spectra of carbon stars available in the low resolution spectral atlas of carbon stars of Barnbaum et al. (1996). However, in order to be able to make a comparison at the same resolution we have acquired a few spectra of comparison stars from Barnbaum et al. (1996) using HFOSC.

In the Morgan-Keenan system (Keenan 1993) carbon stars are divided into C-R, C-N and C-H sequence, with subclasses running to C-R6, C-N6 and C-H6 according to temperature criteria.

The C-N stars have stronger molecular bands and lower surface temperatures than those of C-R stars. C-N stars exhibit strong depression of light in the violet part of the spectrum below about $4500 \AA$ which is believed to be due to scattering by particulate matter. These stars are easily detectable from their characteristic infrared colours. The majority of $\mathrm{C}-\mathrm{N}$ stars show ${ }^{12} \mathrm{C} /{ }^{13} \mathrm{C}$ values more than 30 , ranging nearly to 100 while in C-R stars this ratio ranges from 4 to 9 (Lambert et al. 1986).

\footnotetext{
${ }^{4}$ IRAF (Image Reduction and Analysis Facilty) is distributed by the National Optical Astronomical Observatories, which is operated by the Association for Universities for Research in Astronomy, Inc., under contract to the National Science Foundation.
} 
$\mathrm{C}-\mathrm{R}$ as well as $\mathrm{CH}$ stars have warmer temperatures and blue/violet light is accessible to observation and atmospheric analysis. The s-process element abundances are nearly solar in C-R stars (Dominy 1984) whereas CH stars show significantly enhanced abundances of the s-process elements relative to iron (Lambert et al. 1986, Green \& Margon 1994). However, at low dispersion, the narrow lines are difficult to estimate and essentially do not provide with a strong clue to distinguish C-R stars from $\mathrm{CH}$ stars.

$\mathrm{CH}$ stars form a group of warm stars of equivalent spectral types $\mathrm{G}$ and $\mathrm{K}$ normal giants but show weaker metallic lines. In general, $\mathrm{CH}$ stars are high velocity objects, large radial velocities indicate they belong to the halo population of the Galaxy (McClure 1983, 1984, McClure \& Woodsworth 1990).

As many C-R stars also show quite strong $\mathrm{CH}$ band, the strength and shape of the secondary Pbranch head near $4342 \AA$ is used as a more useful indicator to distinguish them. This is a well-defined feature in $\mathrm{CH}$ stars spectra in contrast to its appearance in C-R stars spectra. Another important feature is the strength of $\mathrm{Ca}$ I at $4226 \AA$ which in case of $\mathrm{CH}$ stars is weakened by the overlying faint bands of the $\mathrm{CH}$ band systems. In $\mathrm{C}-\mathrm{R}$ star this feature is quite strong with band depths deeper than the depth of CN molecular band around $4215 \AA$. Another important feature is the strength of the MerrillSanford (M-S) bands usually ascribed to $\mathrm{SiC}_{2}$, that appear in the wavelength region $4900-4977 \AA$. In general, stars with low ${ }^{12} \mathrm{C} /{ }^{13} \mathrm{C}$ ratios show strong M-S bands. WZ Cas, V Aql and U Cam are a few exceptions which have low ${ }^{13} \mathrm{C}$ but strong $\mathrm{M}-\mathrm{S}$ bands (Barnbaum et al. 1996). $\mathrm{SiC}_{2}$ being a triatomic molecule, $\mathrm{M}-\mathrm{S}$ bands are expected to be the strongest in the coolest stars. $\mathrm{SiC}_{2}$ and $\mathrm{C}_{3}$ have similar molecular structures, and in many $\mathrm{C}$ stars the $\mathrm{C}_{3}$ molecule is believed to be the cause of ultraviolet depression (Lambert et al. 1986 ). M-S bands appear most often in C-J stars, although they are found in some warmer C-N stars. M-S bands are not known to be present in $\mathrm{CH}$ stars.

Based on the above spectral characteristics, we have identified twenty two new $\mathrm{CH}$ star candidates from spectral analysis of about eighty new objects studied recently. These potential $\mathrm{CH}$ star candidates are listed in Table 1, along with those found in our previous studies (Goswami 2005, Goswami et al. 2007, 2010a). All the stars listed in Table 1, are found to fall well within the CH box (Figure 3) defined by Totten et al. (2000).

Strong $\mathrm{C}$-molecular bands but weak $\mathrm{CH}$ band characterize the class of hydrogen deficient carbon stars. In the entire sample of stars studied so far, we have found only one object, HE 1015-2050, to exhibit spectral characteristics of cool HdC star (Goswami et al. 2010b). Figure 2, shows a comparison of the spectrum of this object with other types of carbon stars. Its spectrum matches closely with that of U Aqr. Location of this object in the J-H vs. H-K diagram (figure 3) in the vicinity of U Aqr, HD 182040 and R CrB supports its identification with the group of HdC stars.

\section{Concluding Remarks}

The list of potential $\mathrm{CH}$ star candidates presented here is based on our analysis of low resolution spectra acquired through our on-going observational programs with HCT using HFOSC. Although this observing program has been running for several years, since 2003, the actual observing nights are about two to three nights per observing cycle (in a year there are three observing cycles, each cycle of four months). On many occasions, the allotted nights were not useful for observations due to thick passing clouds and/or strong winds in the direction of our objects. Nonetheless, over the years we have acquired spectra for a sizable fraction from the list of objects we have originally proposed for observation.

The objects identified as potential $\mathrm{CH}$ star candidates will be taken up for a follow-up highresolution spectroscopic study for confirmation of these objects with this class of identification and for a detail study of their chemical composition. Efforts are on to acquire high resolution spectra 


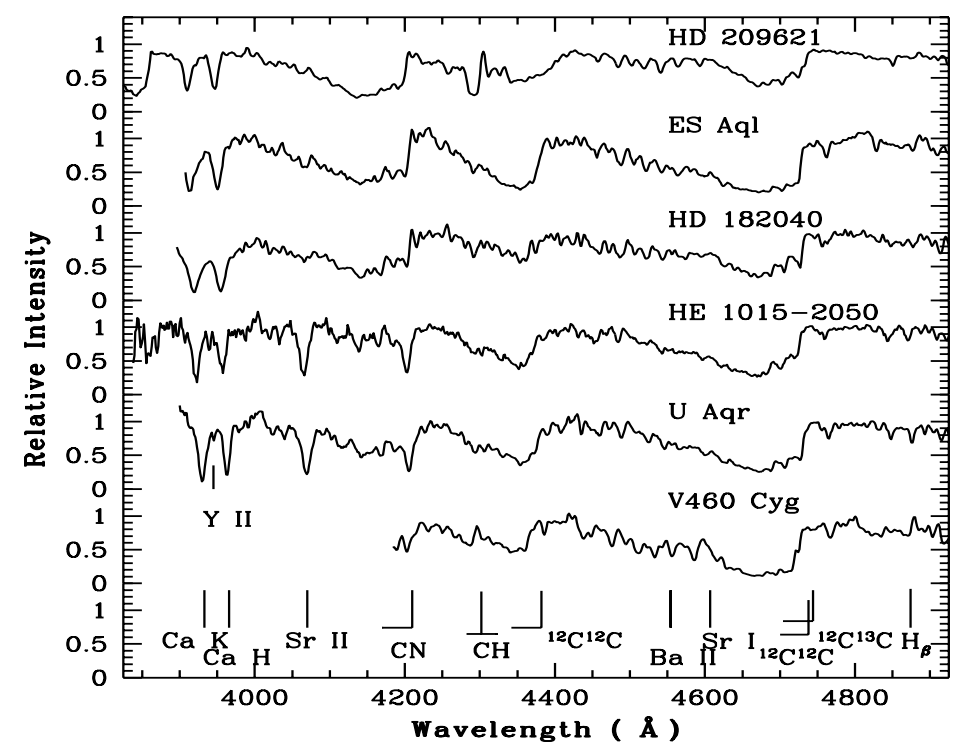

Figure 2: A comparison between the spectrum of HE 1015-2050 with the spectra of V460 Cyg (C-N star), U Aqr, ES Aql (cool HdC stars of RCB type), HD 182040 (a non-variable HdC star), HD 156074 (C-R star), and HD 209621 (CH star) in the wavelength region 3850-4950. . G-band of CH distinctly seen in the $\mathrm{CH}$ and C-R star's spectra are barely detectable in the spectra of HE 1015-2050 and other HdC stars spectra. The large enhancement of Sr II at $4077 \AA$ in the spectrum of U Aqr is easily seen to appear with almost equal strength in the spectrum of HE 1015-2050. Y II line at $3950 \AA$ is detected in the spectra of both HE 1015-2050 and U Aqr. The most striking feature in the spectra of U Aqr and HE 1015-2050 is the strength of the Sr II $\lambda$ 4215 line; this feature is inextricably blended with the nearby strong blue-degraded $(0,1) \mathrm{CN} 4216$ band head in HD 182040. The spectrum of HE 1015-2050 compares closest to the spectrum of U Aqr. (Figure 1, in Goswami et al. 2010b)

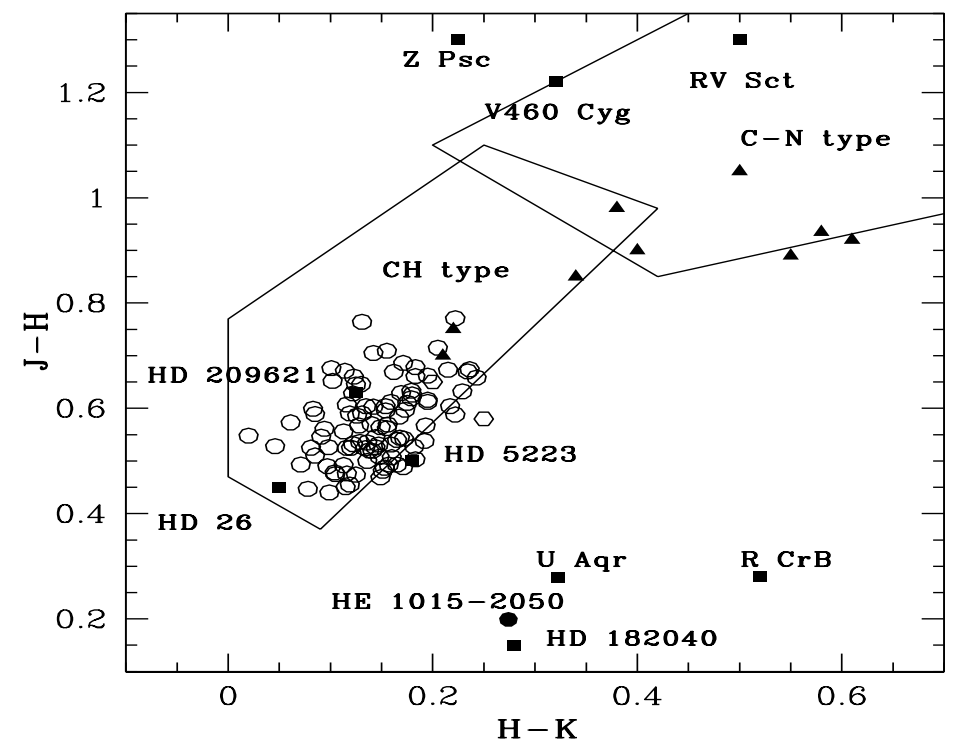

Figure 3: J-H versus $\mathrm{H}-\mathrm{K}$ colour magnitude diagram of the stars listed in Table 1. The $\mathrm{CH}$ stars are represented by open circles. The C-N stars are shown with solid triangles, C-R stars with open hexagons. The position of the HdC star HE 1015-2050 is shown by a filled circle. The thick closed box represents the location of $\mathrm{CH}$ stars and the thin box on the upper right represents the location of C-N stars (Totten et al. 2000) 
for some of these objects, using existing 8-10 m class telescopes as well as HESP attached to HCT. The two main properties, presence or absence of s-process elements and binarity that differentiate early-R stars from $\mathrm{CH}$ stars, can be obtained only through detailed abundance studies that require high resolution spectroscopy, and from long-term radial velocity monitoring. As the objects are quite faint, high resolution spectroscopic studies become arduous and time-consuming. While in this work we have focussed on $\mathrm{CH}$ stars, a detail discussion on objects of other spectral types will be presented in a sequel (under preparation).

\section{Acknowledgements}

AG would like to acknowledge the organizers of $2^{\text {nd }}$ BINA workshop for the local hospitality and the financial support. The funds from BINA projects DST/INT/Belg/P-02 (India) and BL/11/IN07 (Belgium) and funding from DST SERB project No. EMR/2016/005283 are gratefully acknowledged. The author would like to thank Meenakshi and Drisya for help with data reduction.

\section{References}

Aoki W., Ryan S. G., Norris J. E. et al. 2002, ApJ, 580, 1149

Aoki W., Beers T.C., Christlieb N. et al. 2007, ApJ, 655, 492

Barnbaum C., Stone R. P. S., Keenan P. 1996, ApJS, 105, 419

Beers T. C., Christlieb N. 2005, ARA\&A, 43, 531

Bhowmick A., Pandey G., Joshi V. et al. 2018, ApJ, 854, 140

Bonifacio P., Molaro P., Beers T. C. et al. 1998, A\&A, 332, 672

Christlieb N., Green P. J., Wisotzki L. et al. 2001, A\&A, 375, 366

Goswami A. 2005, MNRAS, 359, 531

Goswami A., Aoki W., Beers T. C. et al. 2006 ,MNRAS, 372, 343

Goswami A., Bama P., Shantikumar N. S. et al. 2007, BASI, 35, 339

Goswami A., Karinkuzhi D., Shantikumar N. S. 2010a, MNRAS, 402, 1111

Goswami A., Karinkuzhi D., Shantikumar N. S. 2010b, ApJL, 723, 238

Goswami A., Aoki W., Karinkuzhi D. 2016, MNRAS, 455, 402

Green P. J. \& Margon, B. 1994, ApJ, 423, 723

Hill V., Barbuy B., Spite M. et al. 2000, A\&A, 353, 557

Iben I. J. 1967, ApJ, 147, 624

Keenan Philip C. 1942, ApJ, 96, 101

Keenan Philip C. 1993, PASP, 105, 905

Kumar Y. B., Reddy B. E., Muthumariappan C. et al. 2015, A\&A, 577, 10

Lambert D. L., Gustafsson B., Eriksson K. et al. 1986, ApJS, 62, 373

McClure R. D. 1983, ApJ, 268, 264

McClure R. D. 1984, PASP, 96, 117

McClure R. D., Woodsworth W. 1990, ApJ, 352, 709

McClure R. D., Fletcher J. M., Nemec J. M. 1980, ApJ, 238, 35

Norris J. E., Ryan S. G., Beers T. C. 1997a, ApJ, 488, 350

Norris J. E., Ryan S. G., Beers T. C. 1997b, ApJ, 489, L169

Norris J. E., Ryan S. G., Beers T. C. et al. 2002, ApJ, 569, L107

Ojha D. K., Ghosh S. K., D'Costa S. L. A. et al. 2012, ASInC, Vol 4, 191

Totten E. J., Irwin M. J., Whitelock P. A. 2000, MNRAS, 314, 630

Wallerstein G., Sneden C. 1982, ApJ, 255, 577 
Table 1: Potential $\mathrm{CH}$ star candidates. Quantities labelled with a * upper index are taken from Christlieb et al. (2001b).

\begin{tabular}{|c|c|c|c|c|c|c|c|c|c|c|c|c|}
\hline Star No. & $\mathrm{RA}(2000)$ & DEC (2000) & 1 & $\mathrm{~b}$ & $\mathrm{~B}_{J}^{*}$ & $\mathrm{~V}^{*}$ & $\mathrm{~B}-\mathrm{V}^{*}$ & U-B* & $\mathrm{J}$ & $\mathrm{H}$ & $\mathrm{K}$ & Dt of obs. \\
\hline HE 0008-1712 & 001119.2 & -165534 & 78.58 & -76.21 & 16.5 & 15.2 & 1.78 & 1.64 & 13.630 & 13.069 & 12.975 & 6.12 .2008 \\
\hline HE $0017+0055$ & 002021.6 & +011207 & 106.90 & -60.70 & 12.6 & - & - & - & 9.309 & 8.693 & 8.498 & 15.11 .2003 \\
\hline HE 0038-0024 & 004048.2 & -000805 & 117.09 & -62.89 & 15.4 & 14.4 & 1.86 & 1.67 & 12.433 & 11.768 & 11.573 & 6.11 .2004 \\
\hline HE 0039-2635 & 004139.7 & -261853 & 52.81 & -87.67 & 13.33 & 12.22 & 1.11 & - & 10.571 & 10.115 & 9.996 & 6.12 .2016 \\
\hline HE 0043-2433 & 004543.9 & -241648 & 98.33 & -86.88 & 13.8 & 13.1 & 1.04 & 1.0 & 11.064 & 10.493 & 10.365 & 7.11 .2004 \\
\hline HE 0052-0543 & 005500.0 & -052702 & 125.33 & -68.30 & 16.5 & 15.0 & 1.95 & 1.74 & 12.952 & 12.241 & 12.086 & 12.09 .2008 \\
\hline HE 0100-1619 & 010241.6 & -160301 & 136.76 & -78.61 & 15.9 & 14.7 & 1.54 & 1.28 & 13.114 & 12.537 & 12.476 & 21.11 .2008 \\
\hline HE 0110-0406 & 011237.1 & -035030 & 136.11 & -66.17 & 13.4 & - & - & - & 10.523 & 9.988 & 9.866 & 17.09 .2003 \\
\hline HE 0111-1346 & 011346.5 & -133049 & 145.01 & -75.42 & 13.3 & - & - & - & 10.684 & 10.155 & 10.039 & 7.11 .2004 \\
\hline HE $0113+0110$ & 011552.2 & 012621 & 135.53 & -60.83 & 16.73 & 15.0 & 1.73 & 1.62 & 13.028 & 12.360 & 12.237 & 6.12 .2016 \\
\hline HE 0114-1129 & 011640.3 & -111314 & 144.59 & -72.02 & 15.95 & 14.3 & 1.65 & 1.25 & 12.765 & 12.216 & 12.048 & 23.10 .2005 \\
\hline HE 0136-1831 & 013901.8 & -181643 & 176.49 & -75.91 & 16.9 & 15.6 & 1.72 & 1.43 & 14.216 & 13.679 & 13.532 & 12.09 .2008 \\
\hline HE 0150-2038 & 015310.9 & -202404 & 190.75 & -74.37 & 15.95 & 14.2 & 1.75 & 1.55 & 12.367 & 11.822 & 11.630 & 23.10 .2005 \\
\hline HE 0151-0341 & 015343.3 & -032714 & 157.78 & -62.04 & 14.6 & 13.4 & 1.27 & 0.87 & 11.847 & 11.364 & 11.248 & 7.11 .2004 \\
\hline HE 0206-1916 & 020919.6 & -190156 & 192.69 & -70.38 & 15.07 & 13.9 & 1.17 & 0.63 & 12.243 & 11.765 & 11.660 & 23.10 .2005 \\
\hline HE 0219-1739 & 022141.4 & -172537 & 192.66 & -67.03 & 15.85 & 14.1 & 1.75 & 1.51 & 12.537 & 11.884 & 11.754 & 22.10 .2005 \\
\hline HE 0225-0546 & 022819.4 & -053258 & 174.17 & -58.42 & 16.5 & 15.2 & 1.79 & 1.51 & 13.347 & 12.707 & 12.528 & 6.12 .2008 \\
\hline HE $0237-0835$ & 024013.63 & -082217.8 & 181.93 & -58.16 & 17.27 & 15.60 & 1.67 & 1.42 & 13.706 & 13.136 & 12.987 & 6.12 .2009 \\
\hline HE 0251-2118 & 025342.6 & -210559 & 207.40 & -61.53 & 14.67 & 13.30 & 1.37 & 1.24 & 11.48 & 10.956 & 10.822 & 6.12 .2016 \\
\hline HE 0258-0218 & 030104.89 & -020617.0 & 179.61 & -50.13 & 16.31 & 14.80 & 1.51 & 1.21 & 13.478 & 12.881 & 12.796 & 5.12 .2009 \\
\hline HE 0308-1612 & $03 \quad 1027.1$ & -160041 & 201.12 & -55.96 & 12.5 & - & - & - & 10.027 & 9.475 & 9.331 & 17.09 .2003 \\
\hline HE 0314-0143 & $\begin{array}{llll}03 & 17 & 22.2\end{array}$ & -013237 & 182.98 & -46.69 & 12.7 & - & - & - & 8.993 & 8.222 & 8.000 & 17.09 .2003 \\
\hline HE 0319-0215 & 032146.3 & -020434 & 184.58 & -46.17 & 14.6 & 13.6 & 1.43 & 1.01 & 11.785 & 11.218 & 11.063 & 16.09 .2003 \\
\hline HE 0322-1504 & 032440.1 & -145424 & 201.90 & -52.39 & 15.0 & 13.8 & 1.63 & 1.24 & 12.105 & 11.533 & 11.340 & 6.11 .2004 \\
\hline HE 0420-1037 & 042247.0 & -103026 & 205.04 & -37.71 & 15.2 & 14.7 & 1.38 & 0.99 & 12.341 & 11.815 & 11.695 & 21.11 .2008 \\
\hline HE 0457-1805 & 045943.6 & -180111 & 217.85 & -32.51 & 12.1 & 11.2 & 1.25 & 1.20 & 8.937 & 8.421 & 8.186 & 7.11 .2004 \\
\hline HE $0507-1653$ & 050916.5 & -165005 & 217.54 & -29.96 & 15.6 & 12.4 & 1.06 & 0.68 & 10.883 & 10.430 & 10.315 & 6.11 .2004 \\
\hline HE $0507-1430$ & 051007.6 & -142632 & 215.09 & -28.84 & 16.06 & 14.40 & 1.66 & 1.4 & 12.325 & 11.717 & 11.575 & 20.12.2012 \\
\hline HE 0516-2515 & 051809.4 & -251225 & 227.48 & -30.86 & 15.57 & 13.90 & 1.67 & 1.58 & 11.253 & 10.592 & 10.349 & 6.12 .2016 \\
\hline HE 0518-2322 & 052035.5 & -231914 & 225.62 & -29.74 & 13.7 & - & - & - & 11.151 & 10.672 & 10.568 & 15.11 .2003 \\
\hline HE 1008-0946 & 101122.4 & -100113 & 251.16 & +36.29 & 17.2 & 15.8 & 1.42 & 1.23 & 13.223 & 12.630 & 12.499 & 6.12 .2016 \\
\hline HE $1027-2501$ & 102929.5 & -251716 & 266.68 & +27.42 & 13.9 & 12.7 & 1.73 & 1.51 & - & - & - & 30.03 .2004 \\
\hline HE $1045-1434$ & 104744.1 & -145023 & 263.59 & 38.40 & 15.5 & 14.6 & 1.23 & 0.96 & 12.935 & 12.449 & 12.244 & 9.04 .2007 \\
\hline HE 1051-0112 & 105358.8 & -012815 & 253.52 & 49.79 & 17.0 & 16.0 & 1.44 & 0.94 & 14.347 & 13.794 & 13.703 & 6.12 .2008 \\
\hline HE $1051-0518$ & 105428.8 & -053421 & 257.71 & +46.77 & 14.68 & 13.2 & 1.48 & 1.26 & 11.351 & 10.781 & 10.625 & 30.03 .2005 \\
\hline HE $1056-1855$ & 105912.2 & -191108 & 269.48 & +36.29 & 13.6 & - & - & - & 10.784 & 10.249 & 10.09 & 20.12 .2004 \\
\hline HE 1058-2228 & 110121.8 & -224430 & 272.21 & +33.48 & 14.20 & 13.5 & 1.06 & 0.97 & 12.040 & 11.547 & 11.390 & 19.02 .2006 \\
\hline HE 1102-2142 & 110431.2 & -215829 & 272.52 & 34.50 & 16.0 & 14.9 & 1.44 & 0.98 & 13.275 & 12.714 & 12.601 & 16.03 .2009 \\
\hline HE 1110-0153 & 111302.7 & -020928 & 261.14 & 52.48 & 16.5 & 15.5 & 1.47 & 1.29 & 12.912 & 12.205 & 12.063 & 4.04 .2009 \\
\hline HE 1119-1933 & 112143.5 & -194947 & 275.73 & 38.25 & 12.8 & 14.6 & 1.34 & 0.87 & 13.043 & 12.571 & 12.422 & 18.03.2009 \\
\hline HE 1125-1357 & 112743.0 & -141332 & 274.20 & +43.93 & 15.2 & 14.1 & 1.41 & 1.40 & 11.730 & 11.057 & 10.842 & 12.04 .2004 \\
\hline HE 1120-2122 & 112318.6 & -213833 & 277.12 & 36.77 & 12.9 & - & - & - & 9.573 & 8.902 & 8.788 & 17.03.2009 \\
\hline HE 1123-2031 & 112608.7 & -204819 & 277.44 & 37.80 & 16.8 & 15.8 & 1.33 & 1.19 & 13.513 & 12.940 & 12.800 & 18.03.2009 \\
\hline HE 1142-2601 & 114452.9 & -261829 & 284.84 & 34.21 & 13.9 & 13.0 & 1.28 & 1.07 & 11.218 & 10.675 & 10.539 & 4.04.2009 \\
\hline HE 1145-0002 & 114759.8 & -001919 & 271.30 & +58.60 & 13.5 & 13.6 & 1.48 & 1.49 & 10.911 & 10.240 & 10.006 & 11.04 .2004 \\
\hline HE $1145-1319$ & 114821.4 & -133638 & 280.37 & 46.48 & 16.4 & 15.4 & 1.37 & 1.32 & 13.466 & 12.934 & 12.790 & $\begin{array}{c}17.03 .2009 \\
5.04 .2009\end{array}$ \\
\hline HE 1146-0151 & 114902.3 & -020811 & 273.28 & 57.09 & 14.9 & 14.2 & 0.96 & 0.97 & 12.929 & 12.400 & 12.262 & 16.03.2009 \\
\hline HE 1152-0355 & 115506.1 & -041224 & 277.32 & +55.84 & 13.88 & 11.4 & - & - & 9.339 & 8.665 & 8.429 & 29.01 .2005 \\
\hline HE 1157-1434 & 120011.5 & -145050 & 284.88 & 46.22 & 16.1 & 13.8 & 1.62 & 1.41 & 11.792 & 11.178 & 11.019 & $\begin{array}{l}6.06 .2007 \\
7.06 .2007\end{array}$ \\
\hline HE 1157-0518 & 120018.06 & -053443.1 & 280.33 & +55.03 & 16.27 & 15.120 & 1.14 & 0.97 & 13.418 & 12.917 & 12.846 & 17.05 .2016 \\
\hline HE 1158-0708 & 120049.2 & -072533 & 281.59 & +53.33 & 16.69 & 15.00 & 1.69 & 1.58 & 13.381 & 12.616 & 12.485 & 25.01 .2010 \\
\hline HE 1204-0600 & 120711.6 & -061706 & 283.56 & +54.91 & 14.9 & 14.0 & 1.36 & 1.45 & 11.517 & 10.898 & 10.703 & 11.04 .2004 \\
\hline HE $1205-2539$ & 120808.1 & -255638 & 290.89 & 35.91 & 13.6 & 15.4 & 1.91 & 1.72 & 13.313 & 12.665 & 12.540 & 17.03 .2009 \\
\hline HE $1210-2636$ & 121259.7 & -265322 & 292.43 & 35.19 & 13.8 & 12.6 & 1.65 & 1.52 & - & - & - & 6.05 .2009 \\
\hline HE 1211-0435 & 121412.0 & -045226 & 285.83 & +56.76 & 15.0 & 14.2 & 1.08 & 0.90 & 12.492 & 11.962 & 11.916 & 12.04 .2004 \\
\hline HE 1212-0323 & 121529.1 & -034023 & 285.82 & +57.99 & 16.33 & 15.00 & 1.33 & 1.31 & 13.038 & 12.426 & 12.291 & 30.03 .2017 \\
\hline HE 1221-0651 & 122349.95 & -070754 & 290.9 & +55.09 & 16.13 & 14.80 & 1.33 & 1.36 & 13.037 & 12.423 & 12.307 & 5.03 .2010 \\
\hline HE 1228-0417 & 123112.5 & -043340 & 293.40 & 57.93 & 14.8 & 14.0 & 1.17 & 0.96 & 12.406 & 11.969 & 11.818 & 4.04.2009 \\
\hline HE $1230-0230$ & 123326.4 & -024708 & 293.98 & +59.77 & 14.07 & 12.7 & 1.37 & 1.43 & 10.094 & 9.421 & 9.259 & 29.03.2005 \\
\hline HE 1236-0337 & 123904.59 & -035424.8 & 296.96 & +58.82 & 16.88 & 15.40 & 1.48 & 1.58 & 13.231 & 12.611 & 12.432 & 17.04.2017 \\
\hline HE $1251-2313$ & 125430.92 & -232934.9 & 303.84 & +39.37 & 14.75 & 13.50 & 1.25 & 0.84 & 11.851 & 11.376 & 11.251 & 17.04.2017 \\
\hline HE $1253-1859$ & 125638.4 & -191532 & 304.62 & 43.59 & 13.7 & 12.9 & 1.09 & 1.22 & 10.60 & 9.972 & 9.850 & 16.03.2009 \\
\hline HE 1255-2324 & 125801.17 & -234024.1 & 304.87 & +39.17 & 11.97 & 10.63 & 1.34 & - & 8.659 & 8.171 & 8.00 & 25.01 .2010 \\
\hline HE $1259-2550$ & 130235.40 & 260649.51 & 306.05 & +36.69 & 14.45 & 12.60 & 1.85 & 1.54 & 9.746 & 9.011 & 8.680 & 17.04 .2017 \\
\hline HE 1304-2046 & 130650.1 & -210210 & 307.75 & +41.69 & 15.2 & 14.3 & 1.32 & 1.36 & 11.978 & 11.386 & 11.219 & 30.03 .2004 \\
\hline HE $1305+0007$ & 130803.8 & -000848 & 311.94 & +62.43 & 13.98 & 12.2 & - & - & 10.247 & 9.753 & 9.600 & 29.01 .2005 \\
\hline HE $1305+0132$ & 130817.8 & +011649 & 312.52 & +63.84 & 13.8 & 12.8 & 1.35 & 1.25 & 10.621 & 9.994 & 9.814 & 28.03.2004 \\
\hline HE 1308-1012 & 131110.9 & -102835 & 310.84 & +52.09 & 14.93 & 13.70 & 1.23 & 0.95 & 12.417 & 11.970 & 11.871 & 17.04.2017 \\
\hline HE 1318-1657 & 132119.4 & -171340 & 313.05 & +45.05 & 15.82 & 14.40 & 1.42 & 1.26 & 12.509 & 11.997 & 11.814 & 17.04.2017 \\
\hline HE 1328-0404 & 133111.6 & -041936 & 321.41 & +57.11 & 14.6 & 13.5 & 1.10 & 0.94 & 11.599 & 11.071 & 10.930 & 31.03 .2005 \\
\hline HE $1331-2558$ & 133420.1 & -261338 & 314.78 & 35.65 & 13.9 & 16.0 & 1.53 & 1.10 & 10.998 & 10.394 & 10.240 & $\begin{array}{l}18.03 .2009 \\
17.03 .2009\end{array}$ \\
\hline HE 1331-0247 & 133432.0 & -030230 & 323.61 & +58.09 & 14.32 & 13.0 & 1.32 & 1.31 & 10.998 & 10.394 & 10.240 & 30.03 .2005 \\
\hline HE 1404-0846 & 140655.1 & -090058 & 332.38 & 49.48 & 15.3 & 14.2 & 1.52 & 1.29 & 12.435 & 11.783 & 11.681 & 7.06 .2007 \\
\hline HE $1405-0346$ & 140758.3 & -040103 & 336.53 & 53.78 & 14.7 & 13.5 & 1.68 & 1.28 & 11.608 & 11.068 & 10.903 & 16.03.2009 \\
\hline HE $1410+0213$ & 141306.5 & +015921 & 344.28 & +58.15 & 13.90 & 13.2 & 1.09 & 0.68 & 11.563 & 11.053 & 10.968 & 19.02 .2006 \\
\hline
\end{tabular}


Bulletin de la Société Royale des Sciences de Liège, Vol. 88, Actes de Colloques, 2019, p. 198 - 206

Table 1: (continued)

\begin{tabular}{|c|c|c|c|c|c|c|c|c|c|c|c|c|}
\hline Star No. & $\mathrm{RA}(2000)$ & DEC(2000) & 1 & $\mathrm{~b}$ & $\mathrm{~B}_{J}^{*}$ & $\mathrm{~V}^{*}$ & ${\mathrm{~B}-\mathrm{V}^{*}}^{*}$ & ${\mathrm{U}-\mathrm{B}^{*}}^{*}$ & $\mathrm{~J}$ & $\mathrm{H}$ & $\mathrm{K}$ & Dt of obs. \\
\hline HE $1410-0125$ & 141324.7 & -013954 & 340.63 & 55.09 & - & - & - & - & 10.360 & 9.770 & 9.651 & $\begin{array}{l}16.03 .2009 \\
11.06 .2008\end{array}$ \\
\hline HE $1418+0150$ & 142101.2 & +013718 & 346.80 & 56.66 & 13.70 & - & - & - & 9.988 & 9.356 & 9.127 & 19.02.2006 \\
\hline HE 1425-2052 & 142839.5 & -210605 & 331.40 & 36.33 & 13.6 & 12.7 & 1.27 & 1.29 & 10.043 & 9.446 & 9.273 & 17.03.2009 \\
\hline HE $1429-0551$ & 143231.3 & -060500 & 343.02 & 48.76 & 13.5 & - & - & - & 10.734 & 10.272 & 10.066 & 5.09 .2003 \\
\hline HE $1430+0227$ & 143246.5 & 021444 & 351.53 & 55.24 & 17.59 & 15.9 & 1.69 & 1.75 & 14.024 & 13.482 & 13.310 & 17.05 .2016 \\
\hline HE $1431-0245$ & 143354.2 & -025833 & 346.31 & 51.05 & 16.58 & 15.3 & 1.28 & 1.02 & 13.56 & 13.012 & 12.992 & 19.02 .2017 \\
\hline HE $1431-0755$ & 143432.7 & -080837 & 341.88 & 46.78 & 14.6 & 13.5 & 1.51 & 1.44 & 11.283 & 10.605 & 10.422 & 8.05 .2007 \\
\hline HE $1440-1511$ & 144307.1 & -152348 & 338.73 & 39.57 & 14.5 & 13.5 & 1.13 & 0.87 & 12.238 & 11.757 & 11.606 & 9.05 .2007 \\
\hline HE 1442-0346 & 144502.04 & -035845.5 & 348.70 & 48.53 & 16.67 & 15.4 & 1.27 & 0.93 & 13.749 & 13.249 & 13.113 & 17.04.2017 \\
\hline HE $1446-0112$ & 144902.2 & -012524 & 352.42 & 49.80 & 14.5 & 13.5 & 1.38 & 1.39 & 10.983 & 10.379 & 10.162 & 6.09 .2003 \\
\hline HE $1447+0102$ & 145015.1 & +005015 & 355.22 & 51.22 & 15.6 & 15.0 & 0.9 & 0.14 & 13.207 & 12.760 & 12.682 & 8.04 .2007 \\
\hline HE $1523-1155$ & 152641.0 & -120543 & 351.87 & 35.63 & 14.2 & 13.4 & 1.14 & 0.70 & 11.372 & 10.846 & 10.748 & 29.03 .2004 \\
\hline HE $1524-0210$ & 152656.9 & -022045 & 0.98 & 42.35 & 14.4 & 13.3 & 1.53 & 1.25 & 11.740 & 11.079 & 10.896 & 6.09 .2003 \\
\hline HE $1525-0516$ & 152752.2 & -052704 & 358.11 & 40.10 & 16.8 & 15.8 & 1.29 & 1.14 & 13.972 & 13.479 & 13.314 & $\begin{array}{c}11.06 .2008 \\
7.06 .2007 \\
8.06 .2007\end{array}$ \\
\hline HE 1528-0409 & 153054.3 & -041940 & 359.87 & 40.30 & 15.8 & 15.0 & 1.10 & 0.78 & 12.945 & 12.455 & 12.358 & 29.03.2004 \\
\hline HE 2114-0603 & 211720.8 & -055048 & 45.54 & -34.94 & 16.7 & 15.4 & 1.80 & 1.58 & 12.472 & 11.786 & 11.615 & $\begin{array}{l}11.09 .2008 \\
9.05 .2007\end{array}$ \\
\hline HE 2144-1832 & 214654.7 & -181815 & 34.65 & -46.78 & 12.6 & - & - & - & 8.768 & 8.180 & 7.958 & 16.09 .2003 \\
\hline HE 2145-1715 & 214844.5 & -170103 & 36.63 & -46.73 & 14.2 & 13.2 & 1.39 & 1.18 & 11.032 & 10.356 & 10.255 & 17.09.2003 \\
\hline HE 2153-2225 & 215634.3 & -221125 & 30.16 & -50.16 & 14.91 & 13.5 & 1.41 & 1.15 & 11.446 & 10.847 & 10.764 & 26.07 .2005 \\
\hline HE $2153-2323$ & 215637.6 & -230925 & & & 16.10 & 14.5 & 1.60 & 1.44 & 12.526 & 11.916 & 11.741 & 26.07 .2005 \\
\hline HE 2201-0345 & 220357.5 & -033054 & 56.05 & -43.59 & 15.36 & 14.1 & 1.26 & 0.53 & 12.378 & 11.886 & 11.773 & 22.10 .2005 \\
\hline HE 2207-0930 & 220957.5 & -091606 & 50.27 & -47.96 & 14.4 & 13.1 & 1.82 & 1.40 & 10.527 & 9.812 & 9.607 & 16.09 .2003 \\
\hline HE 2207-1746 & 221037.5 & -173138 & 38.87 & -51.77 & 11.8 & - & - & - & 9.115 & 8.579 & 8.450 & 6.09 .2003 \\
\hline HE 2211-0605 & 221353.5 & -055106 & 55.30 & -46.95 & 16.0 & 15.1 & 1.21 & 1.01 & 13.383 & 12.875 & 12.727 & 24.07 .2008 \\
\hline HE $2218+0127$ & 222126.1 & +014220 & 65.46 & -43.80 & 14.6 & 14.0 & 0.80 & 0.31 & 11.826 & 11.509 & 11.433 & 16.09 .2003 \\
\hline HE 2221-0453 & 222425.7 & -043802 & 59.04 & -48.38 & 14.7 & 13.7 & 1.36 & 1.11 & 11.524 & 10.997 & 10.815 & 17.09 .2003 \\
\hline HE 2228-0137 & 223126.2 & -012142 & 64.49 & -47.70 & 15.8 & 14.7 & 1.56 & 1.20 & 12.301 & 11.715 & 11.589 & $\begin{array}{l}11.09 .2008 \\
25.07 .2009\end{array}$ \\
\hline HE 2234-1017 & 223725.4 & -100219 & 54.90 & -54.12 & 15.64 & 14.2 & 1.44 & 1.43 & 12.420 & 11.913 & 11.753 & 22.10 .2005 \\
\hline HE 2246-1312 & 224926.4 & -125635 & 53.29 & -58.15 & 17.0 & 15.9 & 1.56 & 1.60 & 14.101 & 13.472 & 13.303 & $\begin{array}{l}11.09 .2008 \\
25.07 .2008\end{array}$ \\
\hline HE 2339-0837 & 234159.9 & -082119 & 78.51 & -65.05 & 14.9 & 14.0 & 1.32 & 0.62 & 12.632 & 12.107 & 12.026 & 6.11 .2004 \\
\hline
\end{tabular}

